\title{
Preliminary results of strong ground motion simulation for the Lushan earthquake of 20 April 2013, China
}

\author{
Gengshang Zhu $\cdot$ Zhenguo Zhang $\cdot$ Jian Wen $\cdot$ \\ Wei Zhang $\cdot$ Xiaofei Chen
}

Received: 9 July 2013/Accepted: 6 September 2013/Published online: 30 October 2013

(C) The Seismological Society of China, Institute of Geophysics, China Earthquake Administration, and Springer-Verlag Berlin Heidelberg 2013

\begin{abstract}
The earthquake occurred in Lushan County on 20 April, 2013 caused heavy casualty and economic loss. In order to understand how the seismic energy propagates during this earthquake and how it causes the seismic hazard, we simulated the strong ground motions from a representative kinematic source model by Zhang et al. (Chin J Geophys 56(4):1408-1411, 2013) for this earthquake. To include the topographic effects, we used the curved grids finite difference method by Zhang and Chen (Geophys J Int 167(1):337-353, 2006), Zhang et al. (Geophys J Int 190(1):358-378, 2012) to implement the simulations. Our results indicated that the majority of seismic energy concentrated in the epicentral area and the vicinal Sichuan Basin, causing the XI and VII degree intensity. Due to the strong topographic effects of the mountain, the seismic intensity in the border area across the northeastern of Boxing County to the Lushan County also reached IX degree. Moreover, the strong influence of topography caused the amplifications of ground shaking at the mountain ridge, which is easy to cause landslides. These results are quite similar to those observed in the Wenchuan earthquake of 2008 occurred also in a strong topographic mountain area.
\end{abstract}

Keywords Lushan earthquake - Strong ground motion - Topographic effects - Seismic intensity

G. Zhu $\cdot$ Z. Zhang $\cdot$ J. Wen $\cdot$ W. Zhang $\cdot$ X. Chen $(\bowtie)$

School of Earth and Space Sciences, University of Science and Technology of China, Hefei 230026, China

e-mail: xfchen1@ustc.edu.cn

G. Zhu $\cdot$ Z. Zhang $\cdot$ J. Wen $\cdot$ W. Zhang $\cdot$ X. Chen

Laboratory of Seismology and Physics of Earth's Interior, University of Science and Technology of China, Hefei 230026, China

\section{Introduction}

The Apr 20, 2013 M7.0 earthquake struck the Lushan County of Sichuan Province of China and its surrounding areas (Fig. 1), causing heavy casualties and huge economic losses (Liu et al. 2013). It was reported that this earthquake, occurred at the southwest end of the Longmenshan fault zone, is a thrust faulting event, its epicenter was located in the eastern margin of the Tibetan Plateau, namely the intersection of the Tibetan Plateau and the Sichuan Basin, where geomorphic change is very significant. Therefore, the near field strong ground motion will be strongly affected by the rough topography, i.e., the topographic effect (Geli et al. 1988; Zhang et al. 2008). Shortly after the earthquake, Zhang et al. (2013) obtained the kinematic source model for this earthquake through the teleseismic data inversion. This kinematic source model will not only help us to understand the rupture process and physical mechanism, but also provide fundamental data for computing the strong ground motion and the hazards evaluation of this earthquake. In this study, we adopt the newly developed 3D seismic waves curved grids finite difference method (CG-FDM) which can effectively handle the rough topographic effect (Zhang and Chen 2006; Zhang et al. 2012) and the kinematic source models provided by Zhang et al. (2013) to simulate the strong ground motion, to calculate the corresponding intensity distribution map, and to assess and analyze the hazard distribution.

\section{Methodology and data}

2.1 Numerical simulation method for 3D seismic waves

Earthquake damage is mainly caused by the strong ground motions formed in an earthquake at the free surface. 
Physically, the strong seismic ground motion is the response of seismic waves generated by a rupturing earthquake source. Therefore, if given the kinematic source model as well as the structural model, we can calculate the propagation of seismic waves and its resultant strong ground motion by solving the elastodynamic equations. Since the earthquake occurred at the region with significant topographic variation, the solver we adopted in this study

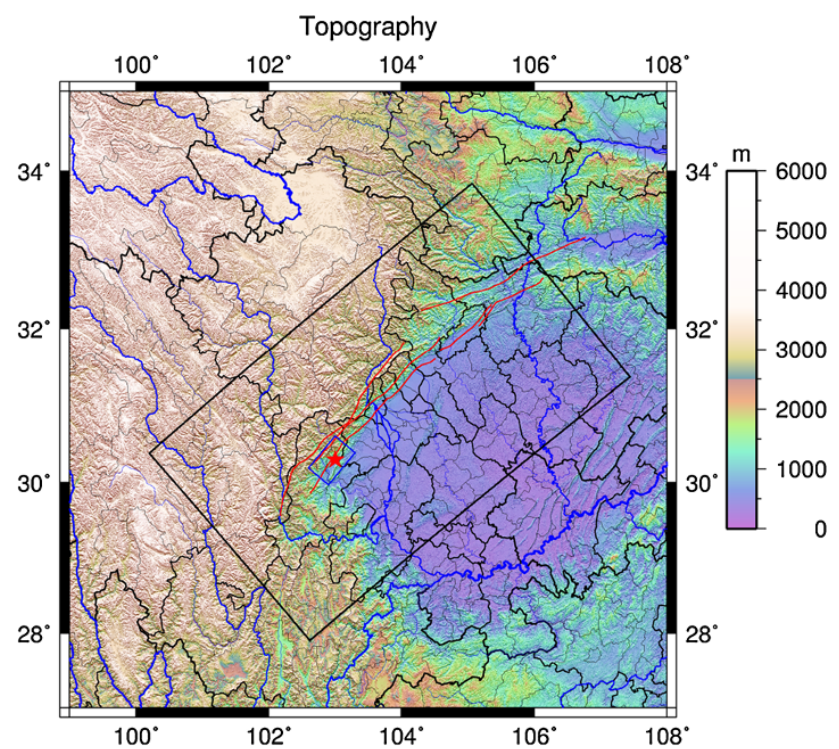

Fig. 1 The map of the region this work concerns. The black box depicts the computational region; the smaller blue box in the middle shows the surface projection of the rupture fault. The red pentagram represents the epicenter of an earthquake and the red lines are faults in Longmenshan fault zone must be able to handle such strong topographic effect. The numerical simulation method adopted in this paper is CGFDM proposed by Zhang and Chen (2006; Zhang et al. 2012). According to the method, curvilinear grids would be generated first as per the arbitrary undulated surface, and then apply the Traction Image method (Zhang and Chen 2006) to handle the free surface condition, so that the method can adequately handle the topographic problem. More details about the method and its application in strong ground motion simulation can be found in Zhang et al. (2008, 2012), Zhang and Chen (2006).

\subsection{Wave-speed model and topography data}

In order to better simulate the effects of seismic waves propagation generated from the source on areas near the fault, especially on the Sichuan Basin, we select a $600 \mathrm{~km} \times 400 \mathrm{~km}$ region to calculate. The calculating depth extends to $50 \mathrm{~km}$ deep. This area with an angle $\mathrm{N}$ $50^{\circ} \mathrm{E}$ (Fig. 1) is approximately parallel with the earthquake fault. In this study, we used a modified global crustal model, CRUST2.0 (Bassin et al. 2000) and a global sediment layer model (SED1.0) (Laske 1997), to approximate the 3D velocity model The topographic model we used in this study is the SRTM elevation data (Digital Elevation Model) with a accuracy of $90 \mathrm{~m}$ (Fig. 2) (Reuter et al. 2007) which is satisfied for our current simulation.

\subsection{Finite fault models}

After the Lushan earthquake, Zhang et al. (2013) quickly inverted the rupture process using the teleseismic data from

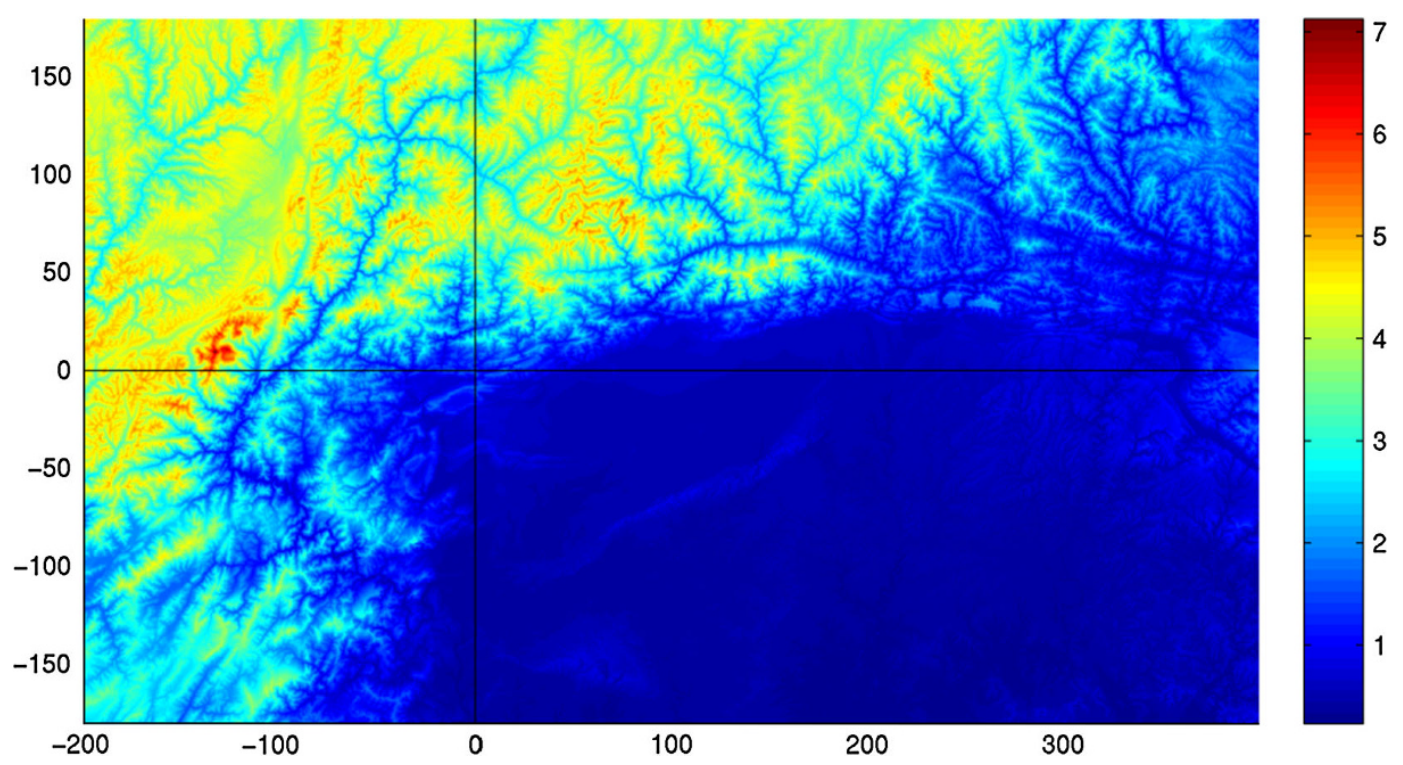

Fig. 2 The topography of computational space. Two black lines are the survey lines. The horizontal line located at $y=0$ and the vertical line located at $x=0$ 
the global seismic network. Zhang et al.'s models show that the earthquake fault is $63 \mathrm{~km}$ in length and $48 \mathrm{~km}$ along the dip, strike angle $219^{\circ}$, dip angle $33^{\circ}$, earthquake moment is about $1.69 \times 10^{19} \mathrm{~N} \mathrm{~m}$, the maximum slip $1.6 \mathrm{~m}$, and focal depth $12.3 \mathrm{~km}$. This finite fault model shows that the earthquake is thrust rupture, and the obvious
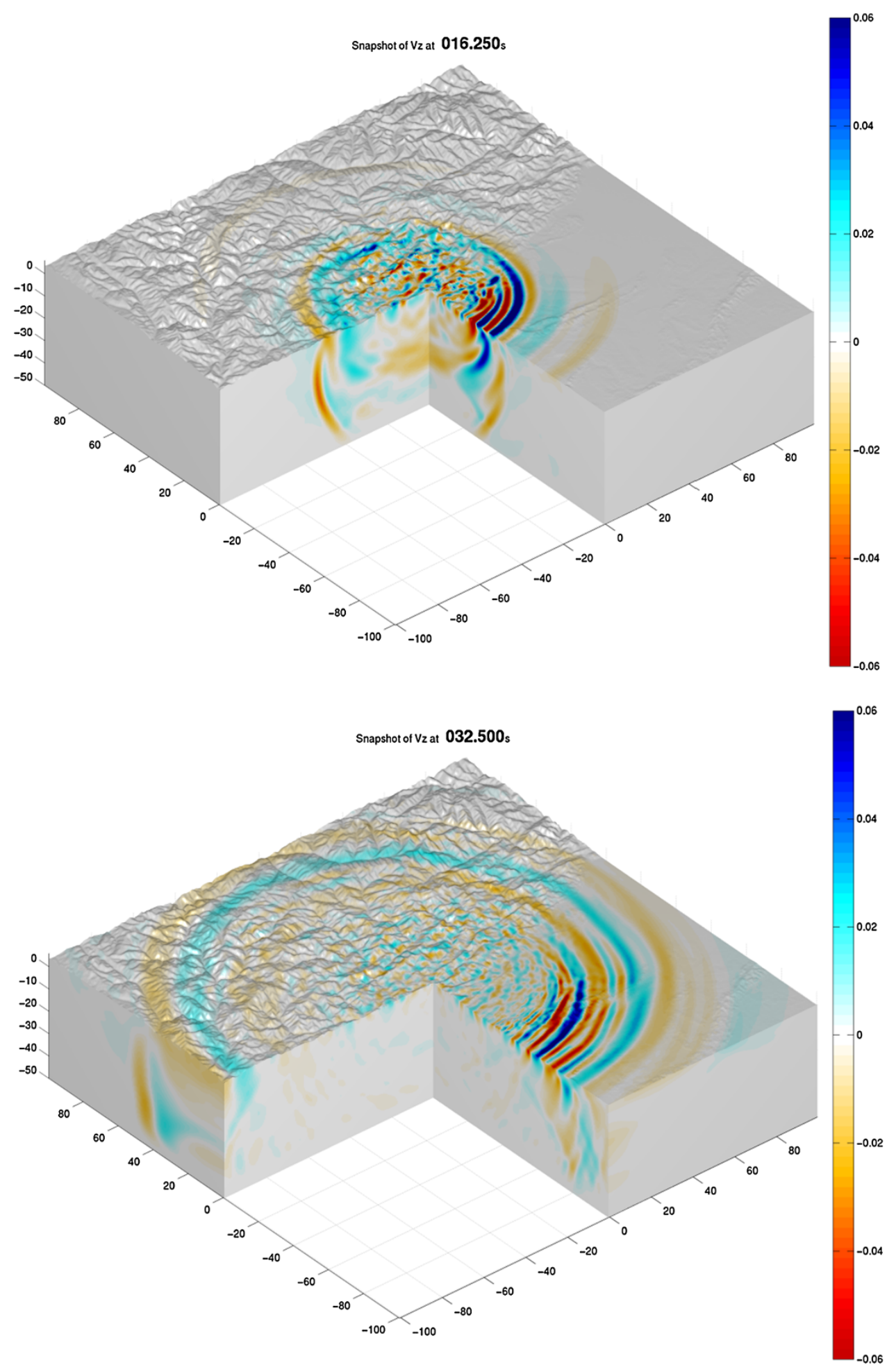

Fig. 3 The snapshots of the vertical component of particle velocity. The time when the snapshot takes is labeled on the top of figures 
rupture directivity and surface rupture is not obvious. In addition, this finite fault model not only give the final slip distribution on the fault plane, but also give the kinematic fault rupture process, i.e., the slip velocity distribution in time and space domain on the fault plane. In this study, we will stimulate the strong ground motion caused by Lushan earthquake based on this finite fault model.

\section{Simulated strong ground motion}

The computational domain is discretized with $1,200 \times 900 \times 180$ grids. The spatial grid size is average $500 \mathrm{~m}$, time step is $0.013 \mathrm{~s}$ and the shortest wavelength is $1,200 \mathrm{~m}$, the highest cutoff frequency is $1.0 \mathrm{~Hz}$, the theoretical calculating seismograms time window is $130 \mathrm{~s}$. To deal with irregular surface, we adopt boundary-conforming curvilinear grid to discretize the computational domain. To ensure accuracy of simulation of seismic waves, the grids near the free surface are condensed, i.e., the vertical space step near the surface is $200 \mathrm{~m}$. Based on the velocity model described above, using the CG-FDM, we simulated the seismic waves with the kinematic source modes provided by Zhang et al. (2013), and the results are shown in Figs. 3, $4,5,6$, and 7 .
3.1 The simulated wave-field and seismograms

The simulated wave-fields are showed in Fig. 3. The results show strong amplifications of seismic waves in the basin area as well as in some small localized patches in the fault area. The former amplification in basin area is due to the reverberation effect of seismic waves in the thick lowvelocity basin, the later amplifications in the small localized patches in the epicentral mountain area are due to the topographic effects also observed in the 2008 Wenchuan earthquake (Zhang et al. 2008). The main radiated seismic energy of Lushan earthquake propagates mainly toward the East with slightly South direction. Figures 4 and 5 show the calculated seismograms at the two survey lines $(y=0$ and $x=0$ ) on the ground surface. It can be seen from the results that in the survey line $y=0$ the amplitudes of seismic waves in side of $x>0$ are apparently larger than those of $x<0$, while the amplitudes of seismic waves in side of $y<0$ is larger than those of $y>0$. This is due to the amplification effects of Sichuan basin.

\subsection{Peak ground velocity and seismic intensity map}

Figure 6 shows the intensity maps which made by the PGV distributions as per the Chinese intensity scale (Chen et al.
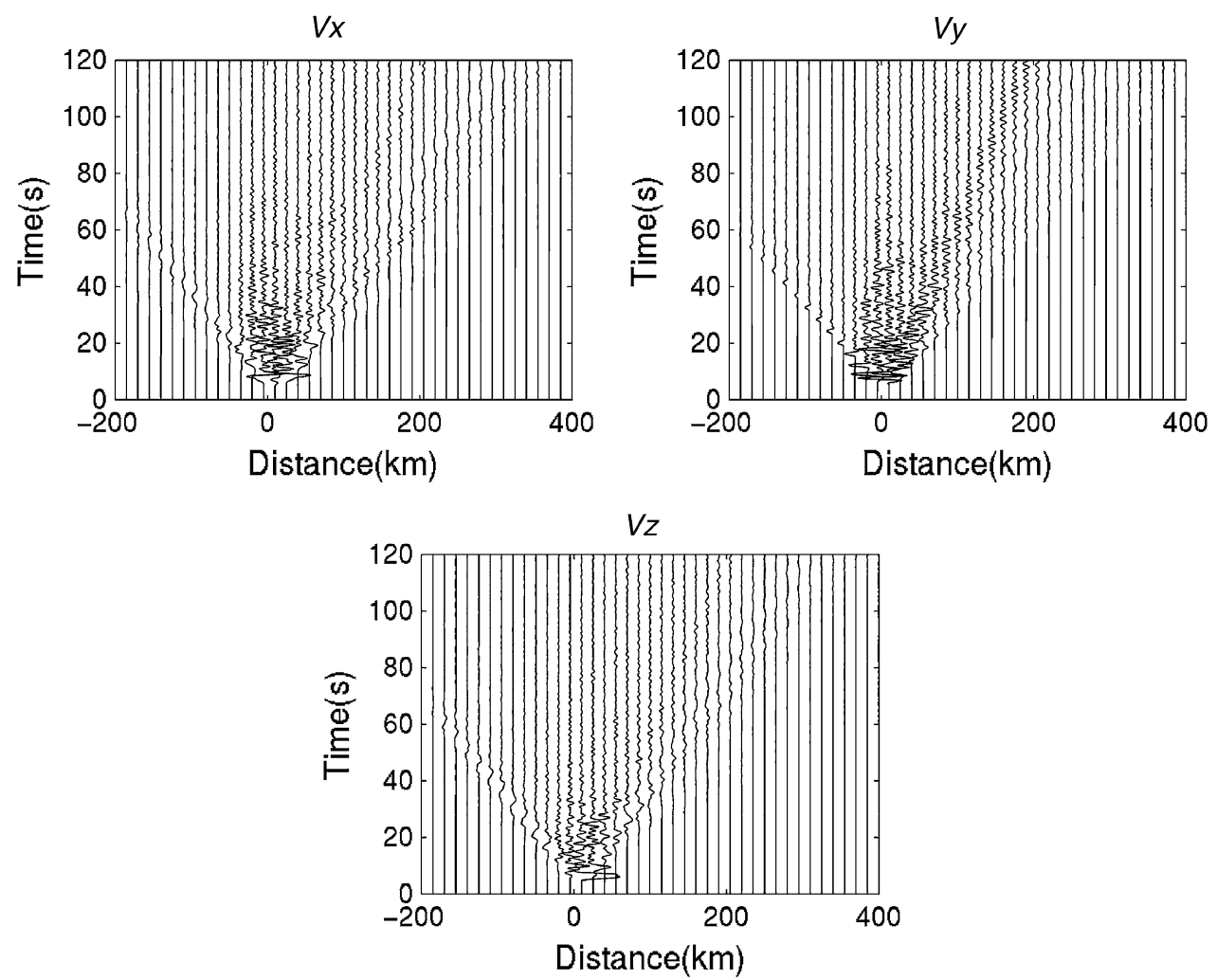

Fig. 4 The synthetical seismograms (three components) in the horizontal survey line of Fig. 2 

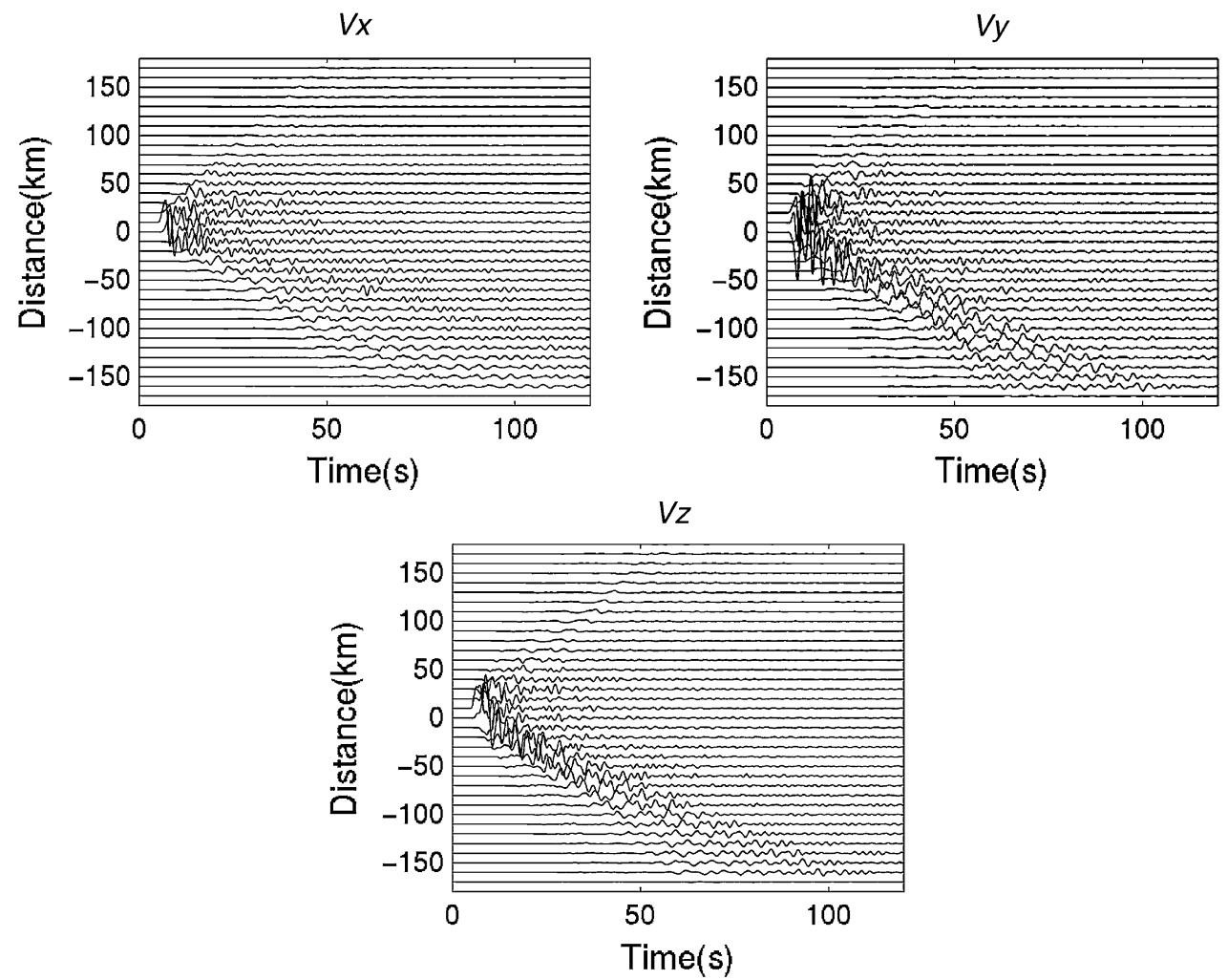

Fig. 5 The synthetical seismograms (three components) in the vertical survey line of Fig. 2

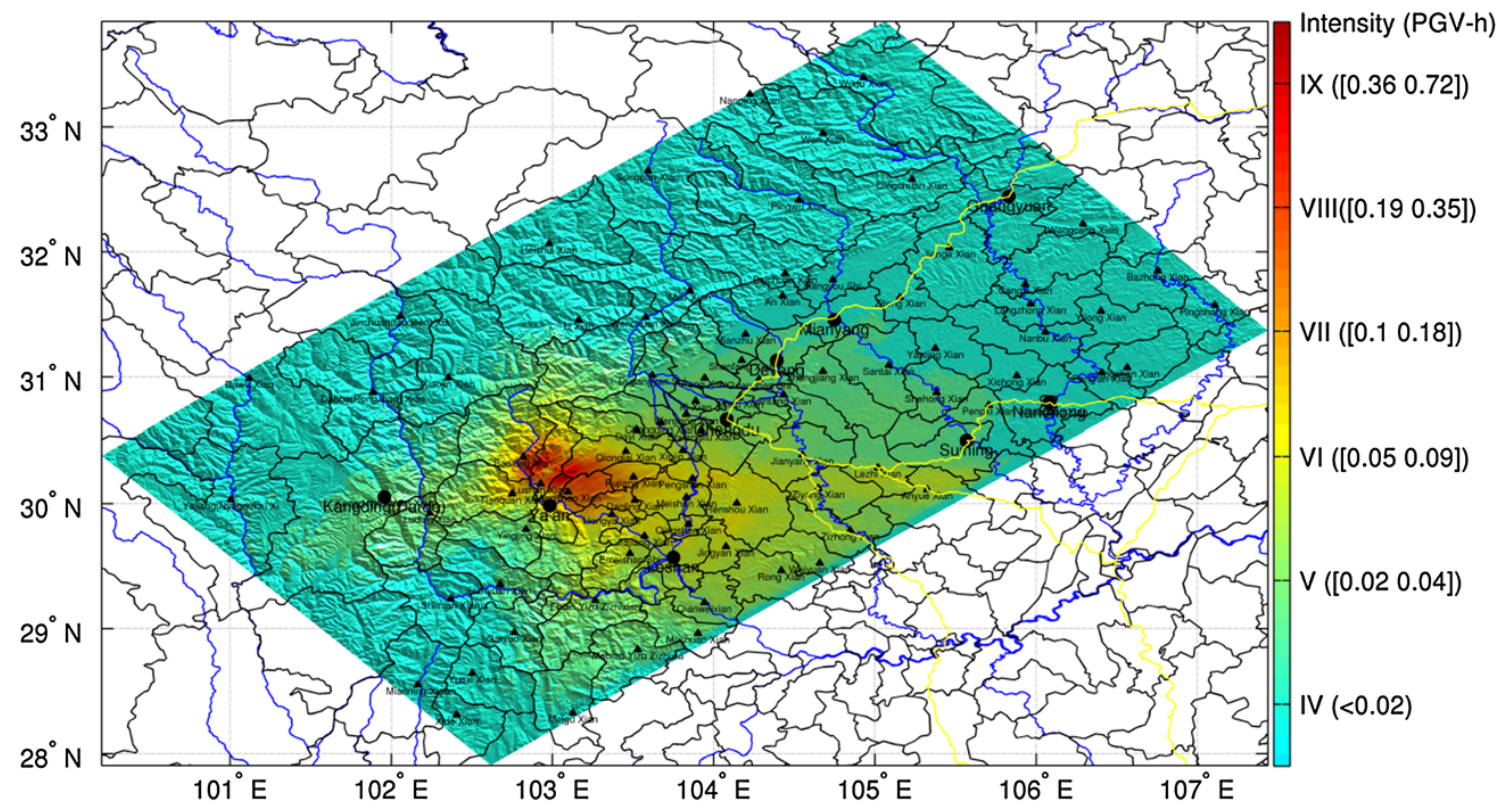

Fig. 6 The predicted seismic intensity map, the color scale shows the level of seismic intensity and the corresponding range of horizontal PGV

1999). The maps show that the maximum intensity is IX degree. The IX degree region is not only located in the near-fault zone but also in some crests which located in the border region of Baoxing county and Lushan county as showed in Fig. 7. The IX degree zones include the projection area of the ruptured fault (hanging wall effect), and 

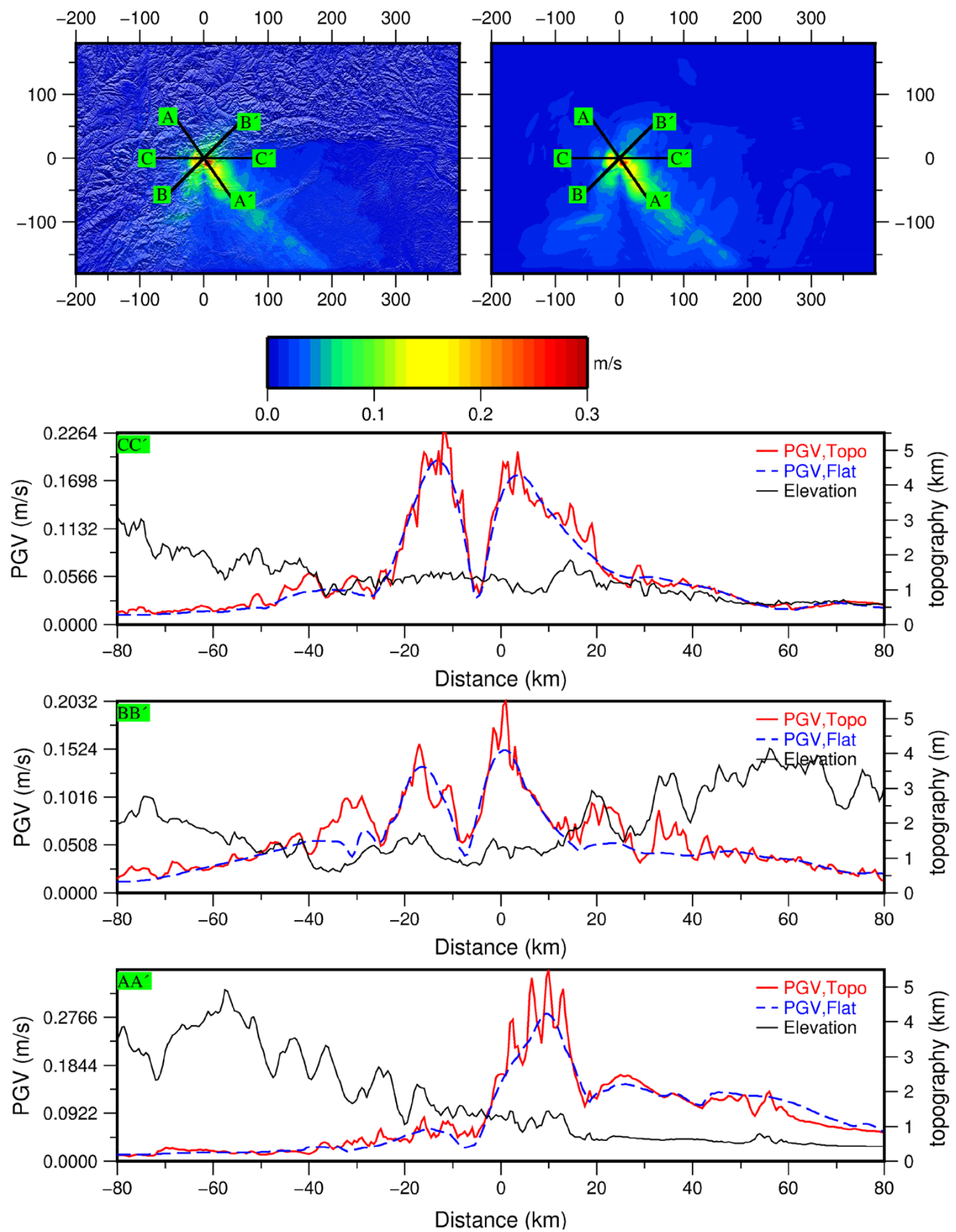

Fig. 7 The comparison of the PGV with real topography surface model (top, left) and the PGV with the flat surface model (top, right). Three profiles selected in the following comparisons are represented by solid lines. For the detail comparisons (see the text), PGV from simulations with real topography and with flat surface are illustrated by red solid line and blue dashed line separately. The elevation is plotted by black line

some mountain ridges in the border zone of Baoxing county and Lushan county due to the topographic effects. The relatively broad basin region with intensity VII-VIII degree is due to the basin amplification effects.

\section{Discussion and conclusions}

To assess the topography effects, we will compare the distribution of PGV between the model with real 
topography surface and with flat surface. In the case of flat surface, we set the topography elevation to the mean elevation to make the paths of wave propagation be nearly the same to the case with real topography. The comparison of PGV in the fault-parallel component between the case of model with real topography surface (left) and model with flat surface (right) could be found in Fig. 7. The same color scale is chose to make the comparison more clear.

We select three profiles $\left(\mathrm{AA}^{\prime}, \mathrm{BB}^{\prime}, \mathrm{CC}^{\prime}\right)$ to explain the effect of topography on strong ground motion and the profiles are all across the epicenter. The comparison of three profiles is plotted separately in Fig. 7. In each comparison, the red line implies the PGV of real topography, and the blue dotted line implies the PGV of flat surface model. The elevations are implied in the black lines. From the Fig. 7, we find four characteristics: (1) the PGV with the flat surface is smoothly distribution, in contrast, the PGV of real topography surface model is modulated by surface topography; (2) the PGV with the flat surface model is smaller than the PGV with the surface topography because of the topography amplification effects; (3) the PGV values of real topography surface model is modulated by surface topography, in other words, the PGV on the peaks of the mountain ranges is larger than the PGV in the valleys;(4) the features of the topography effects is more obvious on the mountain ranges above the fault zone than those in the mountain ranges far away from the fault zone.

Based on the kinematic source models provided by Zhang et al. (2013), we simulate the strong ground motion of Lushan earthquake by using the CG-FDM (Zhang and Chen 2006; Zhang et al. 2012) with the SRTM digital topographic model and the Crust2.0 and SED1.0 velocity model, and obtain the seismic intensity map. Our results show that, the main seismic energy of Lushan earthquake concentrated on fault zone area and the Sichuan Basin, and the corresponding seismic intensities reach up to IX degree. It is noticed that the seismic intensity in the border zone of Baoxing country and Lushan country also is also up to IX degree, reflecting a strong near field topographic effect.
Such topographic effect was also observed in the strong ground motion simulation of the Wenchuan earthquake (Zhang et al. 2008).

Acknowledgments We are deeply grateful to Dr. Zhang Yong for providing us kinematic source model. The authors also thank recommendations from the two anonymous and the editor for improving this work. This research was supported by the National Natural Science Foundation under Grant 41090290.

\section{References}

Bassin C, Laske G, Masters G (2000) The current limits of resolution for surface wave tomography in North America. EOS Trans Am Geophys Union 81:F897

Chen D.-S., Shi Z.-L., Xu Z.-H. et al. (1999) China seismic intensity scale. General administration of quality supervision, inspection, and quarantine of P.R.C. http://www.dccdnc.ac.cn/html/zcfg/ gfxwj2.jsp (in Chinese)

Geli L, Bard PY, Jullien B (1988) The effect of topography on earthquake ground motion: a review and new result. Bull Seismol Soc Am 78(1):42-63

Laske G, Masters G (1997) A global digital map of sediment thickness. EOS Trans Am Geophys Union 78:F483

Liu J, Yi G-X, Zhang Z-W et al (2013) Introduction to the Lushan, Sichuan M7.0 earthquake on 20, April 2013. Chin J Geophys 56(4):1404-1407. doi:10.6038/cjg20130434 (in Chinese)

Reuter H-I, Nelson A, Jarvis A (2007) An evaluation of void filling interpolation methods for SRTM data. Int J Geogr Inf Sci 21(9):983-1008

Zhang W, Chen X-F (2006) Traction image method for irregular free surface boundaries in finite difference seismic wave simulation. Geophys J Int 167(1):337-353

Zhang W, Shen Y, Chen X-F (2008) Numerical simulation of strong ground motion for the ms 8.0 Wenchuan earthquake of 12 May 2008. Sci China Ser D Earth Sci 51(12):1673-1982

Zhang W, Zhang Z-G, Chen X-F (2012) Three-dimensional elastic wave numerical modelling in the presence of surface topography by a collocated-grid finite-difference method on curvilinear grids. Geophys J Int 190(1):358-378

Zhang Y, Xu L-S, Chen Y-T (2013) Rupture process of the Lushan 4.20 earthquake and preliminary analysis on the disaster-causing mechanism. Chin J Geophys 56(4):1408-1411. doi:10.6038/ cjg20130435 (in Chinese) 